\title{
Intermode Breather Solitons in Optical Microresonators
}

\author{
Hairun Guo, Erwan Lucas, Martin H. P. Pfeiffer, Maxim Karpov, Miles Anderson, \\ Junqiu Liu, Michael Geiselmann, John D. Jost, and Tobias J. Kippenberg ${ }^{*}$ \\ École Polytechnique Fédérale de Lausanne (EPFL), Lausanne CH-1015, Switzerland
}

(Received 7 June 2017; published 6 December 2017)

\begin{abstract}
Dissipative solitons can be found in a variety of systems resulting from the double balance between dispersion and nonlinearity, as well as gain and loss. Recently, they have been observed to spontaneously form in Kerr nonlinear microresonators driven by a continuous wave laser, providing a compact source of coherent optical frequency combs. As optical microresonators are commonly multimode, intermode interactions, which give rise to avoided mode crossings, frequently occur and can alter the soliton properties. Recent works have shown that avoided mode crossings cause the soliton to acquire a singlemode dispersive wave, a recoil in the spectrum, or lead to soliton decay. Here, we show that avoided mode crossings can also trigger the formation of breather solitons, solitons that undergo a periodic evolution in their amplitude and duration. This new breather soliton, referred to as an intermode breather soliton, occurs within a laser detuning range where conventionally stationary (i.e., stable) dissipative Kerr solitons are expected. We experimentally demonstrate the phenomenon in two microresonator platforms (crystalline magnesium fluoride and photonic chip-based silicon nitride microresonators) and theoretically describe the dynamics based on a pair of coupled Lugiato-Lefever equations. We show that the breathing is associated with a periodic energy exchange between the soliton and a second optical mode family, a behavior that can be modeled by a response function acting on dissipative solitons described by the Lugiato-Lefever model. The observation of breathing dynamics in the conventionally stable soliton regime is relevant to applications in metrology such as low-noise microwave generation, frequency synthesis, or spectroscopy.
\end{abstract}

DOI: 10.1103/PhysRevX.7.041055

Subject Areas: Nonlinear Dynamics, Optics, Photonics

\section{INTRODUCTION}

Dissipative solitons are self-localized structures resulting from the double balance of dispersion by nonlinearity and dissipation by a driving force, and have been observed in a variety of fields, such as plasma physics, atomic physics, chemistry, and biology [1]. In optics, dissipative solitons describe a range of both spatial and temporal selforganization phenomena leading to stable self-similar structures in optical cavities, known as spatial or temporal cavity dissipative solitons. Beyond such stable states, cavity dissipative solitons can also exhibit oscillatory or periodic evolutions such as pulsating dynamics [2], soliton molecular vibrations $[3,4]$, and temporal breather solitons [5-7]. In particular, temporal breather solitons undergo periodic oscillations in their amplitude and duration. First studied in the context of the Fermi-Pasta-Ulam recurrence [8], they have been observed in a wide range of optical systems, including nonlinear fiber optics [9], optical modelocked lasers [10], nonlinear fiber cavities [5], and recently

\footnotetext{
*tobias.kippenberg@epfl.ch
}

Published by the American Physical Society under the terms of the Creative Commons Attribution 4.0 International license. Further distribution of this work must maintain attribution to the author(s) and the published article's title, journal citation, and DOI. in the context of dissipative Kerr solitons (DKS) in optical microresonators [11-13]. DKS in Kerr nonlinear microresonators, first observed in crystalline microresonators [14], can spontaneously form when pumping the microresonator with an external continuous-wave (cw) laser [14,15], and have emerged as an ideal platform for the study of dissipative soliton physics. Likewise, DKS constitute a way to generate coherent optical frequency combs. In recent years, DKS have been extensively studied and have been observed in a variety of microresonator platforms [16-21]. Their spectrally broadband and fully coherent nature, exhibiting a large repetition rate (in the $\mathrm{GHz}$ to $\mathrm{THz}$ range), have been successfully applied in a number of applications, including spectroscopy $[22,23]$, counting of optical frequencies [24,25], coherent communication [26], low-noise microwave generation [17], dual comb based distance measurements [27,28], and have provided even a route to a fully integrated optical frequency synthesizer [29]. In addition to stable DKS, breather solitons in microresonators have recently been observed and studied [11-13]. In those studies, the breathing phenomenon corresponds to an intrinsic dynamical instability of dissipative Kerr cavity systems [5-7,30] described by a standard Lugiato-Lefever equation (LLE) model (or equivalently a set of coupled-mode equations) [31-34], which exists near the low-detuning boundary of the soliton existence domain. For stable single and multiple DKS, the detuning-dependent existence range has also been experimentally analyzed and 
compared to theory [35], and is modified by the thermal effect enabling the number of DKS in a given state to be reduced deterministically [36].

In this work, we demonstrate a novel type of dissipative breather soliton triggered by avoided mode crossings (abbreviated as AMX hereafter) that are ubiquitous phenomena in multimode microresonators. Indeed, most microresonator platforms today are multimode either inherently or as a result of waveguide dispersion engineering. For instance, to support DKS, thick (typically > $800 \mathrm{~nm}$ at $1550 \mathrm{~nm}$ wavelength) silicon nitride $\left(\mathrm{Si}_{3} \mathrm{~N}_{4}\right)$ waveguide-based microresonators $[37,38]$ are required to obtain the necessary anomalous group velocity dispersion. Therefore, they are prone to having intermode coupling. AMX then describe a situation when two modes exhibit a similar resonance frequency and, through coupling, a pair of symmetric and asymmetric modes is formed with new resonance frequencies [39]. It has been shown that AMX can prevent soliton formation or, if sufficiently weak and rare, lead to local alterations in the spectrum of the solitonbased frequency comb [40]. In particular, AMX can lead to the formation of dispersive waves $[41,42]$ that induce a soliton spectral recoil, and in recent work have been shown to enable the reduction of the repetition rate noise [43] by balancing the Raman self-frequency shift $[16,44,45]$. Yet, to date, little is known about soliton dynamics in the presence of intermode coupling.

Here, we discover that DKS can feature breathing by a periodic energy exchange between the soliton and another mode family. We term this phenomenon an intermode breather soliton. Remarkably, the breathing dynamics occur in a regime where the conventional LLE predicts stable cavity solitons $[14,30,46]$. We observe such intermode interactions in two microresonator platforms, i.e., a magnesium fluoride $\left(\mathrm{MgF}_{2}\right)$ crystalline resonator and a $\mathrm{Si}_{3} \mathrm{~N}_{4}$ waveguide-based microresonator, and confirm it with numerical simulations based on a set of coupled LLEs $[43,47]$. Our observations not only contribute to the physics of DKS with a new panel of soliton instability, but are also critical for applications ranging from self-referenced soliton combs for frequency metrology [15,24], to the generation of low-noise microwaves [17].

\section{THEORY AND SIMULATION}

Intermode interactions between microresonator mode families consist of both linear and nonlinear couplings. The linear coupling can be described by coupled-mode theory $[48,49]$, while the nonlinear coupling mainly refers to the cross-phase modulation in Kerr cavities. In a weakcoupling limit, one can assume that DKS remain supported in a primary mode family $(\mathcal{P})$ that is linearly coupled to a second crossing mode family $(\mathcal{C})$. The integrated dispersion of the primary soliton-supporting mode family is defined as $D_{\text {int }}^{(\mathcal{P})}(\mu)=\omega_{\mu}^{(\mathcal{P})}-\omega_{0}^{(\mathcal{P})}-\mu D_{1}^{(\mathcal{P})}$ [where $\omega_{\mu}^{(\mathcal{P})}$ indicates the resonance frequency over the relative mode index $\mu$, $\mu=0$ is the central pumped mode, and $D_{1}^{(\mathcal{P})} / 2 \pi$ is the free spectral range (FSR)], whereas relative to this frequency grid, the crossing mode family has the frequency given by $\Delta(\mu)=\omega_{0}^{(\mathcal{C})}-\omega_{0}^{(\mathcal{P})}+\mu\left(D_{1}^{(\mathcal{C})}-D_{1}^{(\mathcal{P})}\right) \quad[$ Fig. $1(\mathrm{a})]$. Therefore, an AMX occurs around the mode where $D_{\text {int }}^{(\mathcal{P})}(\mu) \approx \Delta(\mu)$. Moreover, a soliton-based frequency comb is fully coherent with equally spaced frequency components (i.e., dispersionless), such that it appears as a straight line in the frame of $D_{\text {int }}^{(\mathcal{P})}(\mu)$, see Fig. 1(a), and is supported in a continuous range of laser detuning $2 \pi \delta=$ $\omega_{0}^{(\mathcal{P})}-\omega_{p}>0$ [i.e., the pump $\left(\omega_{p}\right)$ is necessarily red detuned from the central mode]. Therefore, at a given detuning $\delta$, the phase-matching condition between the soliton to a wave in the crossing mode $\mu_{c}$ is given by

$$
2 \pi \delta+\Delta\left(\mu_{c}\right) \approx 0 .
$$

The dynamics of DKS in the presence of intermode interactions can be fully explained by two sets of coupledmode equations (or equivalently two coupled LLEs) including both the linear coupling and the crossphase modulation [43,47], as detailed in Appendix A. Alternatively, effects of the linear coupling can be approximated by a suitable response incorporated into a single set of coupled-mode equations (or a single LLE framework), which accounts for soliton dynamics in the primary mode family. In a "co-traveling" frame (i.e., a frame that is cotraveling with the soliton waveform centered at the pump frequency), such a single LLE-like model is written as

$$
\begin{aligned}
\frac{\partial \tilde{A}_{\mu}^{(\mathcal{P})}(t)}{\partial t}= & \left(-\frac{\kappa^{(\mathcal{P})}}{2}+i(2 \pi \delta)+i D_{\mathrm{int}}^{(\mathcal{P})}(\mu)\right) \tilde{A}_{\mu}^{(\mathcal{P})}+\tilde{R}_{c}(\mu) \tilde{A}_{\mu}^{(P)} \\
& -i g \mathcal{F}\left[\left|A^{(P)}\right|^{2} A^{(P)}\right]_{\mu}+\delta_{\mu 0}^{\prime} \sqrt{\kappa_{\mathrm{ex}}} s_{\mathrm{in}}
\end{aligned}
$$

where $\tilde{A}_{\mu}^{(\mathcal{P})}$ and $A^{(\mathcal{P})}$ are the spectral and temporal envelopes of DKS, respectively [related via $A^{(\mathcal{P})}(t)=$ $\left.\sum_{\mu} \tilde{A}_{\mu}^{(\mathcal{P})} e^{-i \mu D_{1}^{(\mathcal{P})} t}\right], \kappa^{(\mathcal{P})}$ is the loss rate of the solitonsupporting primary mode family, $g$ is the single-photoninduced Kerr frequency shift (see Appendix A for the full definition), $\kappa_{\mathrm{ex}}$ is the external (waveguide induced) coupling rate, $\left|s_{\text {in }}\right|^{2}$ denotes the pump power, $\delta_{\mu 0}^{\prime}$ is the Kronecker delta, and $\mathcal{F}[]_{\mu}$ represents the $\mu$ th frequency component of the Fourier series. We derive an intermode response accounting for the linear coupling:

$$
\tilde{R}_{c}(\mu)=\frac{G^{2}}{\mathcal{D}(\mu)}\left[e^{(\mathcal{D}(\mu) t)}+1\right]
$$

where $\mathcal{D}(\mu)=-\left(\kappa^{(\mathcal{C})} / 2\right)+i[2 \pi \delta+\Delta(\mu)]$ contains the phase-matching condition. The response bandwidth is defined by the loss rate of the crossing mode family 

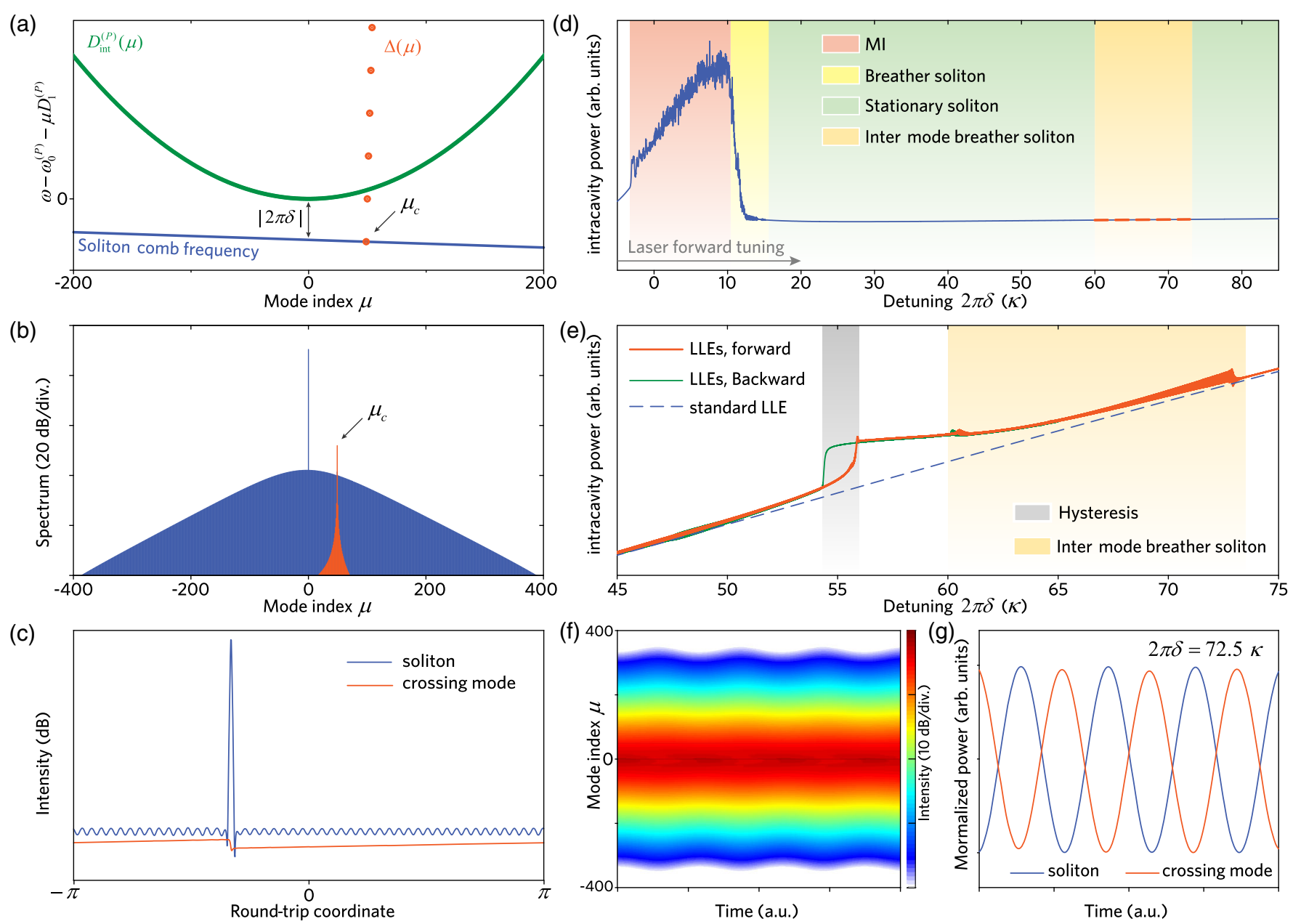

FIG. 1. Numerical simulation of an intermode breather soliton in a Kerr microresonator. (a) Integrated dispersion of a (primary) soliton-supporting mode family (green dots) in which the parabolic profile indicates the anomalous group velocity dispersion in this mode family. In the same frame, the crossing mode family shows a sloped profile corresponding to $\Delta(\mu)$ (red dots). Once the solitonbased frequency comb is formed with a detuning $(\delta)$, the soliton comb frequency represents nearly a constant $(-2 \pi \delta)$ (blue line), implying equal distance between comb teeth. The slight slope corresponds to a change in the FSR (compared to $D_{1}^{(P)} / 2 \pi$ ) as a result of the soliton central frequency shift caused either by a dispersive-wave-induced soliton spectral recoil or by the Raman self-frequency shift. Thus, the phase matching between the soliton and the wave in the crossing mode $\left(\mu_{c}\right)$ is $\Delta\left(\mu_{c}\right)+2 \pi \delta \approx 0$. (b) Simulated singlesoliton-based frequency comb in the primary mode family (blue lines) and a narrow band waveform in the crossing mode family (orange lines). (c) Intracavity field patterns in both mode families. (d) Intracavity power trace over the laser detuning, based on a standard LLE model in the absence of intermode interactions (blue line). Three stages, modulation instability (MI) regime (red area), breather soliton (yellow area), and stationary soliton (green area), are marked as typical transitions of frequency comb states in the generation process. The intermode breather soliton exists in the region where stationary DKS would be expected (orange area). (e) DKS in the presence of intermode interactions (based on the coupled LLEs model) show a different behavior, including a hysteretic power transition (gray area) and an oscillatory behavior (orange area). (f) Spectral envelope evolution of a single intermode breather soliton. (g) Out-of-phase oscillations in the energy of the soliton and the crossing mode waveform.

$\left(\kappa^{(\mathcal{C})}\right)$ and the response amplitude is scaled by the strength of the linear coupling $(G)$ between the primary and the crossing mode families. Compared to the full model, such a single LLE-like model can equivalently reproduce the soliton dynamics in the presence of linear intermode interactions. Nevertheless, if there exists more than one crossing mode fulfilling the phase-matching condition [Eq. (1)], the LLE-like model can be flexibly extended by including several intermode responses [Eq. (3)].
We next perform simulations based on the coupled LLEs. Figure 1 shows a simulation of a single-solitonbased frequency comb in the presence of intermode interactions. The soliton comb envelope in the primary mode family remains overall a sech ${ }^{2}$ profile. In the crossing mode family a spectrally narrow band waveform is generated, contributing a single, power-enhanced wave (in the mode $\mu_{c}$ ) to the overall soliton comb spectrum [Fig. 1(b)], which is phase matched to the cavity soliton. This 
power-enhanced comb tooth then behaves similarly to a dispersive wave [43] causing a temporal oscillation in the intracavity field pattern [Fig. 1(c)] and inducing a soliton recoil such that the comb envelope is shifted in a spectral direction opposite to that of the dispersive wave. The temporal intracavity field in the crossing mode family is almost continuously distributed corresponding to the power-enhanced wave, but features a power step induced by the soliton via the cross-phase modulation [Fig. 1(c)].

Usually, in the absence of intermode interactions, DKS are known to exist within a continuous range of the laser detuning, called the soliton existence range, in which the soliton is stationary and the soliton power smoothly evolves over the change of detuning [green shaded area in Fig. 1(d)]. This is because the soliton peak intensity, as well as the pulse duration, is scaled by the detuning $(\delta)$ [14,50]. At the lower boundary of the soliton existence range is the intrinsic breathing state that has an oscillatory power trace (yellow shaded area). Here, however, we observe strikingly different soliton dynamics within its stationary existence range [highlighted as the orange shaded area in Fig. 1(d)]. First, when tuning $\delta$, the intracavity power shows an abrupt transition mainly contributed by the formation of the power-enhanced wave localized in the mode $\mu_{c}$ of the crossing mode family [Fig. 1(e)]. Comparing traces in both forward (increased detuning) and backward (decreased detuning) scans of the detuning, a hysteretic behavior on the soliton power is revealed, which is in agreement with a recently proposed theory [43]: it is a result of the single-mode dispersive-wave-induced soliton spectral recoil that leads to a modification of the phasematching criterion and entails a power bistability with respect to the detuning.

Second, and most interestingly, we discover breathing dynamics from intermode interactions, as indicated by oscillations and increased amplitude jitter in the power trace in close vicinity of the bistability region of the comb teeth power [Fig. 1(e)], which we term an intermode breather soliton. A periodic spectrum evolution of such a breather soliton is also observed in the simulation [Fig. 1(f)], which reveals an oscillation of the soliton pulse duration-an essential feature of breather solitons. Moreover, a closer analysis reveals an energy exchange regime between the soliton and the waveform in the crossing mode family, where out-of-phase power oscillations are observed [Fig. 1(g)].

Such phenomena exactly reflect a general property of dissipative solitons that hysteresis usually exists around a transition of the system state [51,52], here from the stable soliton state to breathing under intermode interactions.

\section{EXPERIMENTAL RESULTS}

The novel intermode breather solitons are experimentally obtained in two microresonator platforms: $\mathrm{MgF}_{2}$ crystalline microresonators and chip-scale $\mathrm{Si}_{3} \mathrm{~N}_{4}$ microresonators. In both platforms, a single cavity soliton can be deterministically generated in the cavity by using the laser tuning method $[14,36]$. In general, as a solution of the LLE model, DKS are known to have a characteristic squared hyperbolic-secant temporal intensity profile, whose optical spectrum also corresponds to a squared hyperbolic-secant envelope. Previous studies [14,16] demonstrated this relation and other works presented alternative means of identifying a soliton state allowing the bypass of direct time-domain analysis (e.g., frequency-resolved optical gating measurement; cf. Ref. [14]). Here, we base our study on frequency domain quantities that hold the advantage of fast acquisition and high dynamic range: the soliton repetition rate is measured from the radio frequency (rf) beat note of the frequency comb teeth spacing, and the pulse duration is estimated via the comb bandwidth. In particular, the soliton state can also be identified and characterized by measuring the system's response to a pump modulation [36], which reveals the effective laser-to-resonance detuning $\delta$-a key parameter for cavity DKS. Stabilizing the effective detuning enables us to directly compare experimentally generated DKS to the theoretically predicted stability chart [35] and, especially in this work, identify deviations induced by intermode interactions. The system response also constitutes a fingerprint of the soliton state, by showing a characteristic double resonance feature known as the $\mathcal{C}$ resonance, which indicates the effective detuning, and the soliton-related $\mathcal{S}$ resonance [36].

Here, we focus on the soliton dynamics when the effective detuning is well within the stationary soliton existence range such that the $\mathcal{C}$ and $\mathcal{S}$ resonances are far separated. This is to avoid the intrinsic breathing dynamics which appears at a much lower detuning value [13]. In a $\mathrm{MgF}_{2}$ microresonator, we generate a single-soliton-based frequency comb (FSR $\sim 14 \mathrm{GHz}$ ) that has an overall $\mathrm{sech}^{2}$ shape spectral envelope, but features several powerenhanced comb teeth due to the phase matching to the cavity soliton [Fig. 2(a)]. While sweeping the laser frequency, such that the effective detuning is continuously changed but remains within the soliton existence range, we observe the appearance of a breathing feature [Figs. 2(b) and 2(c)] in the form of sidebands on the rf beat note of the comb with a fundamental breathing frequency of $\sim 3 \mathrm{MHz}$. Concomitantly, in the system's response, a strong-amplitude tone appears at the same frequency and close to the $\mathcal{S}$ resonance. Such characteristics emerge in a narrow detuning range $(\delta \sim 12.8 \mathrm{MHz})$ and are missing when the soliton is outside of this range. We note that the breathing frequency slightly shifts with the detuning, which in the current case increases with an increase of the detuning [Fig. 2(c)].

Similarly, in a $\mathrm{Si}_{3} \mathrm{~N}_{4}$ microresonator, a single-solitonbased frequency comb (with FSR $\sim 1 \mathrm{THz}$ ) is also generated [Fig. 3(a)] where intermode interactions result in a dispersive wavelike spectral wave packet rather than a 


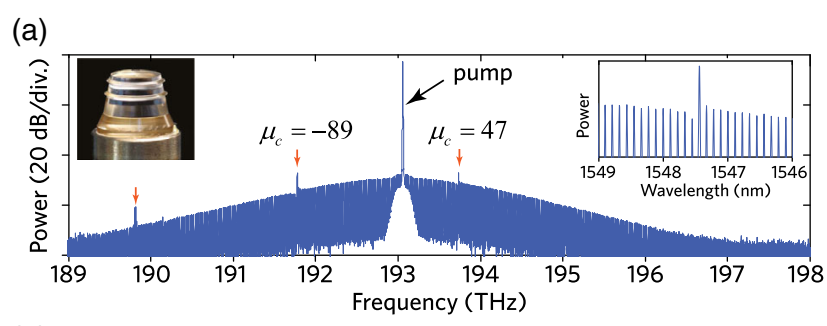

(b)
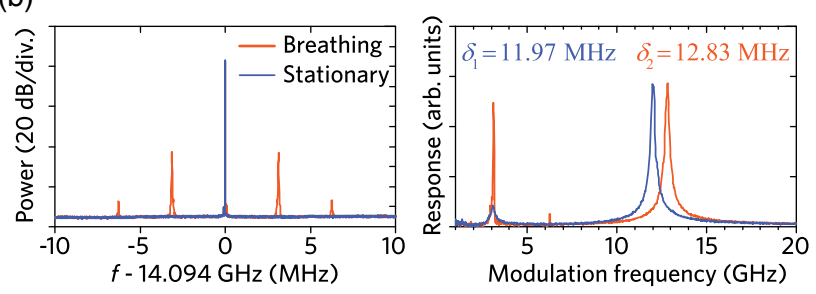

(c)

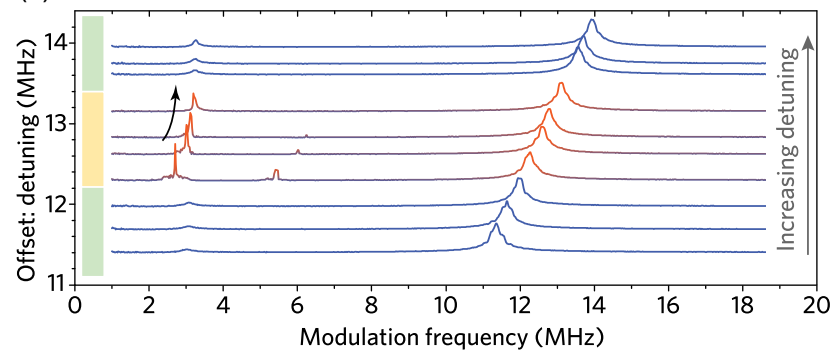

FIG. 2. Observation of an intermode breather soliton in a $\mathrm{MgF}_{2}$ crystalline microresonator ( $\sim 14 \mathrm{GHz}$ FSR). (a) Experimentally generated single-soliton-based frequency comb exhibiting spikes (i.e., enhanced power in comb teeth) from intermode interactions. Inset: Zoomed-in spectrum around the mode $\mu_{c}=47$. (b) The beat note measurements (left) and system response measurements (right) for both the stationary soliton state and the intermode breathing state. (c) Measured evolution of the system's response when increasing the laser detuning. The soliton features breathing in the detuning range $12.3-13.2 \mathrm{MHz}$ where a strong frequency tone (and harmonics) is observed in the system's response close to the $\mathcal{S}$ resonance. The color bar marks the region of breathing which is within the stationary soliton existence range. The curved arrow marks the shift of the breathing frequency with an increase of the detuning.

single, power-enhanced wave, implying that the bandwidth of the intermode response $\tilde{R}_{c}$ is large (compared to the comb teeth spacing). Sweeping the effective detuning in the stationary soliton existence range, frequency tones appear in both the rf spectrum and the system's response, in which a fundamental breathing frequency of $\sim 1 \mathrm{GHz}$ is identified [Fig. 3(b)]. Shift of the breathing frequency over the detuning is also observed.

In experiments, we also monitor the power of each power-enhanced wave, i.e., the modes $\mu_{c}=-7$ and $\mu_{c}=-25$. We observe not only an abrupt power transition of these waves over the detuning, but also the hysteresis in the transition [Fig. 3(c)]. Such a phenomenon, as previously only theoretically predicted (referred to as the hysteresis in the power of a single-mode dispersive wave) [43], is experimentally observed here in both modes, when (a)

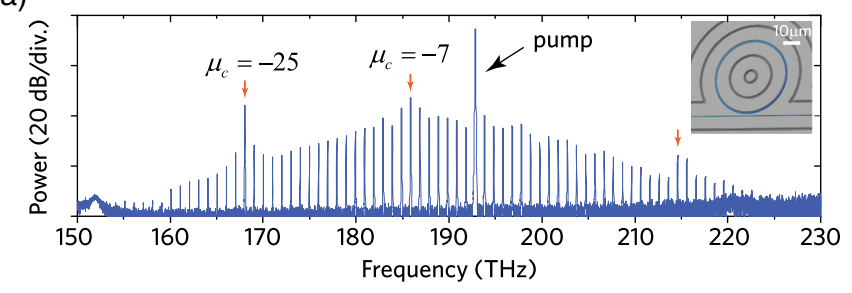

(b)

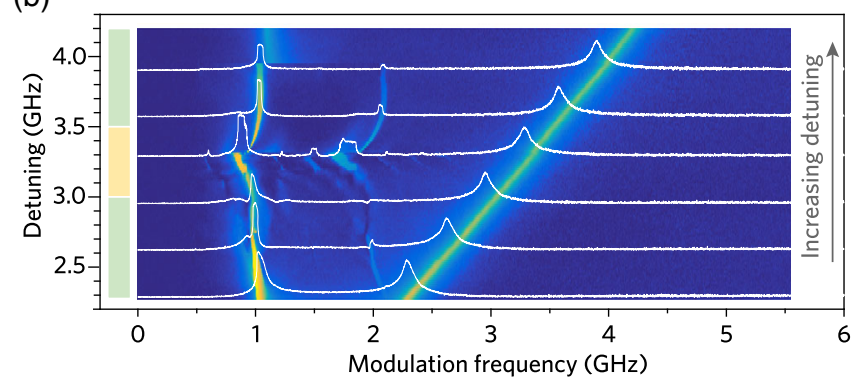

(c)

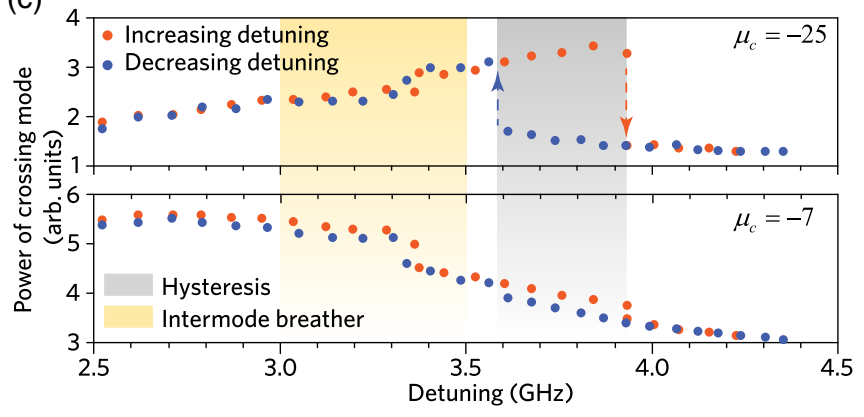

FIG. 3. Observation of an intermode breather soliton in a chipscale $\mathrm{Si}_{3} \mathrm{~N}_{4}$ microresonator and hysteresis in the comb profile. (a) Experimentally generated single-soliton-based frequency comb in a silicon nitride resonator (with a $\sim 1 \mathrm{THz}$ FSR) featuring spikes (i.e., power-enhanced comb teeth) from intermode interactions. (b) Measured evolution of the system response when increasing the laser detuning. The intermode breathing is in the detuning range 2.4-3.9 GHz. The color bar marks the region of breathing. (c) Measured power of single comb mode $\mu_{c}=-7$ and $\mu_{c}=-25$ for both forward and backward laser detuning. The measurements reveal a clear hysteresis of the enhanced comb teeth power (gray area).

the laser tuning direction is reversed. Abrupt power transition is also observed in the $\mathrm{MgF}_{2}$ resonator, but the hysteretic behavior is weak, probably due to the weaker nonlinear effect than $\mathrm{Si}_{3} \mathrm{~N}_{4}$ (cf. Appendix C).

Interestingly, we notice that the breathing frequency of $\sim 3 \mathrm{MHz}$ in the $\mathrm{MgF}_{2}$ resonator and $\sim 1 \mathrm{GHz}$ in the $\mathrm{Si}_{3} \mathrm{~N}_{4}$ microresonator are both similar to that of an intrinsic breathing soliton [13]. In between these two types of microresonators, the 3-orders-of-magnitude difference in the breathing frequency can be related to the difference in the resonator's $Q$ factors, respectively, a $\mathcal{O}(0.1) \mathrm{MHz}$ resonance linewidth in the $\mathrm{MgF}_{2}$ resonator and a $\mathcal{O}(100) \mathrm{MHz}$ linewidth in the $\mathrm{Si}_{3} \mathrm{~N}_{4}$ microresonator.

Furthermore, we experimentally demonstrate an energy exchange regime in the intermode breather soliton (see 
(a)

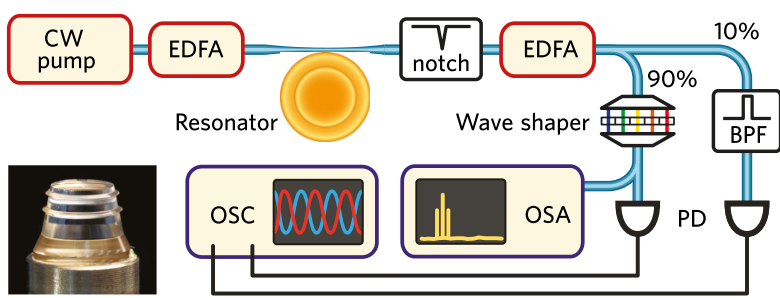

(b)

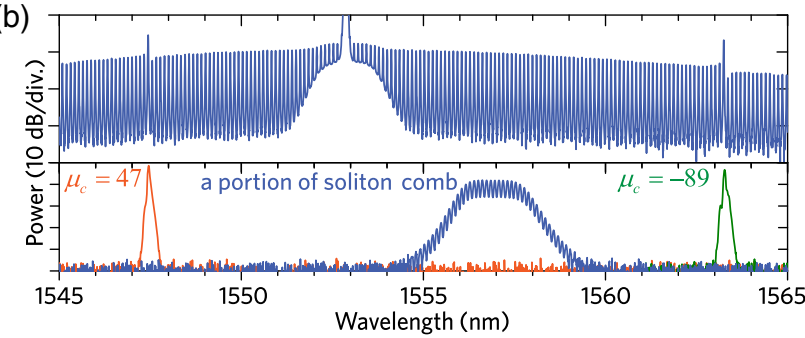

(c)

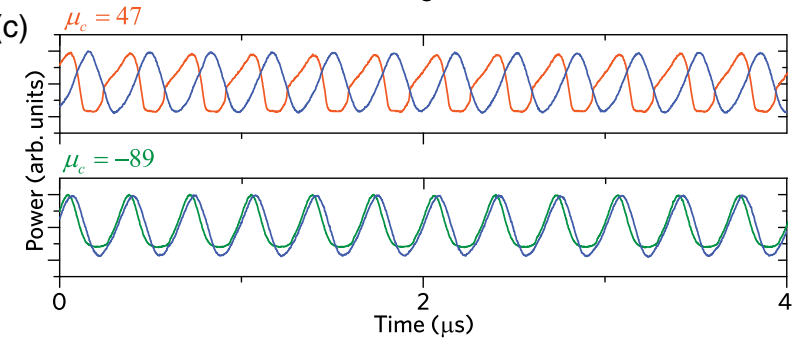

FIG. 4. Observation of periodic energy exchange in an intermode breather soliton state. (a) Schematic of the experimental setup. EDFA, erbium-doped fiber amplifier; BPF, bandpass filter; PD, photodetector; OSC, oscilloscope; OSA, optical spectral analyzer. (b) Generated single-soliton-based frequency comb in the $\mathrm{MgF}_{2}$ microresonator (upper) and filtered components (lower) by using a wave shaper and by a bandpass filter. (c) Recorded fast power evolution of a single filtered comb mode, $\mu_{c}=47$ (red) and $\mu_{c}=$ -89 (green) compared to a "regular" filtered portion of the soliton spectrum (blue line). The power in $\mu_{c}=47$ shows an out-of-phase oscillation to the soliton (upper), while the power in $\mu_{c}=-89$ shows an in-phase oscillation (lower).

Fig. 4), by measuring both the power in the phase-matched mode and the power of the soliton. Using a wave shaper [operational wavelength $1527-1600 \mathrm{~nm}$, see sketch in Fig. 4(a)], power-enhanced waves in modes $\mu_{c}=47$ and $\mu_{c}=-89$ of the $\mathrm{MgF}_{2}$ resonator comb are selectively filtered [Fig. 4(b)]. Their oscillatory power traces (reflecting the breathing) are compared to that of a portion of the single-soliton-based frequency comb obtained through a bandpass filter. In this way, we observe that the power in the mode $\mu_{c}=47$ shows an out-of-phase oscillation with respect to the soliton power, while the power in the mode $\mu_{c}=-89$ oscillates in phase [see Fig. 4(c)]. These observations are in excellent agreement with our simulations [Fig. 1(g)], implying that the wave mainly localized in the mode $\mu_{c}=47$ of the crossing mode family is actively interacting with the soliton in the primary mode family, and is causing the breathing through energy exchange. In contrast, power-enhanced waves that are oscillating in phase do not lead to breathing dynamics.

\section{CONCLUSION}

In conclusion, we investigate novel breathing dynamics of cavity dissipative Kerr solitons in the presence of intermode interactions originating from AMXs in multimode microresonators. AMXs lead to the formation of power-enhanced comb teeth in the soliton-based frequency comb spectrum through a phase-matching condition. The power-enhanced wave, mainly localized in the crossing mode family, not only exhibits a hysteresis in the power evolution over the laser detuning, but can also feature an energy exchange with the soliton in the primary mode family, which is understood as the origin of the soliton breathing dynamic. Such an intermode breather soliton is distinct from the intrinsic breather soliton as it is observed to exist in a detuning range where the regular LLE predicts stationary DKS. We observe the intermode breather soliton in both a $\mathrm{MgF}_{2}$ crystalline resonator and a chip-based $\mathrm{Si}_{3} \mathrm{~N}_{4}$ microresonator and perform simulations that qualitatively confirm our experimental observations. Our results provide a new insight into the dynamics of cavity dissipative solitons by revealing an energy exchange regime that can generally exist in multimode cavities. Equally important, they are highly relevant to application as intermode breather solitons indicate a new panel of soliton instability which is detrimental to low-noise microwave generation, for example. Moreover, we can further confirm experimentally that intermode interactions can induce soliton decay as well as soliton switching. The abrupt power transition as well as the breathing within the stationary soliton existence range can perturb states with multiple DKS, leading to a decrease in the number of solitons inside the resonator (i.e., soliton switching).

The code and data used to produce the plots within this paper are available at [53]. All other data used in this study are available from the corresponding authors upon reasonable request.

\section{ACKNOWLEDGMENTS}

This work was supported by Contract No. W31P4Q-14-C0050 from the Defense Advanced Research Projects Agency (DARPA), Defense Sciences Office (DSO); by the Air Force Office of Scientific Research, Air Force Material Command, USAF under Award No. FA9550-15-1-0099; by funding from the European Union's Horizon 2020; and by the Swiss National Science Foundation under Grant Agreement No. 161573; H. G. acknowledges the support from research and innovation programme under Marie Sklodowska-Curie IF Grant Agreement No. 709249. E. L. and M. K. acknowledge the support from the European Space Technology Centre with ESA Contracts No. 4000116145/16/NL/MH/ GM and No. 4000118777/16/NL/GM. M. K. acknowledges the support from the European Union's FP7 programme under Marie Sklodowska-Curie Initial Training Network Grant Agreement No. 607493. M. G. acknowledges support 
from the Hasler foundation and support from the "EPFL Fellows" fellowship program co-funded by Marie Curie, FP7 Grant Agreement No. 291771. $\mathrm{Si}_{3} \mathrm{~N}_{4}$ samples were fabricated and grown in the Center of MicroNanoTechnology (CMi) at EPFL.

\section{APPENDIX A: COUPLED LUGIATO-LEFEVER EQUATIONS AND SINGLE EQUATION MODEL}

In a Kerr cavity system with two mode families, i.e., the soliton-supporting primary $(\mathcal{P})$ mode family and a crossing
$(\mathcal{C})$ mode family, intermode interactions can be described by two sets of coupled-mode equations including both the linear coupling and cross-phase modulation (i.e., the nonlinear coupling) [43,47], which are equivalent to two Lugiato-Lefever equations, each describing the waveform dynamics in the corresponding mode family. In a frame that is co-traveling with the waveform in the primary mode family (free spectral range $D_{1}^{(\mathcal{P})} / 2 \pi$ ) centered at the pump frequency $\left(\omega_{p}\right)$, the two sets of coupled-mode equations are written as

$$
\begin{aligned}
\frac{\partial \tilde{A}_{\mu}^{(\mathcal{P})}(t)}{\partial t}= & \delta_{\mu 0}^{\prime} \sqrt{\kappa_{\mathrm{ex}}} s_{\mathrm{in}}+\left(-\frac{\kappa^{(\mathcal{P})}}{2}+i(2 \pi \delta)+i D_{\mathrm{int}}^{(\mathcal{P})}(\mu)\right) \tilde{A}_{\mu}^{(\mathcal{P})}-i G \tilde{A}_{\mu}^{(\mathcal{C})} \\
& -i g^{(\mathcal{P})} \mathcal{F}\left[\left|A^{(\mathcal{P})}\right|^{2} A^{(\mathcal{P})}\right]_{\mu}-i g^{(\mathcal{C}-P)} \mathcal{F}\left[2\left|A^{(\mathcal{P})}\right|^{2} A^{(\mathcal{P})}\right]_{\mu}, \\
\frac{\partial \tilde{A}_{\mu}^{(\mathcal{C})}(t)}{\partial t}= & \left(-\frac{\kappa^{(\mathcal{C})}}{2}+i(2 \pi \delta)+i\left[D_{\mathrm{int}}^{(\mathcal{C})}(\mu)+\Delta(\mu)\right]\right) \tilde{A}_{\mu}^{(\mathcal{C})}-i G \tilde{A}_{\mu}^{(\mathcal{P})} \\
& -i g^{(\mathcal{C})} \mathcal{F}\left[\left|A^{(\mathcal{C})}\right|^{2} A^{(\mathcal{C})}\right]_{\mu}-i g^{(\mathcal{C}-P)} \mathcal{F}\left[2\left|A^{(\mathcal{P})}\right|^{2} A^{(\mathcal{C})}\right]_{\mu},
\end{aligned}
$$

where $\tilde{A}_{\mu}^{(\mathcal{P})}$ and $\tilde{A}_{\mu}^{(\mathcal{C})}$ represent the spectral field envelope in the primary and the crossing mode families, respectively, $A^{(\mathcal{P})}$ and $A^{(\mathcal{C})}$ are the temporal amplitudes of the intracavity field, $\kappa^{(\mathcal{P})}$ and $\kappa^{(\mathcal{C})}$ indicate the cavity decay rates, and $2 \pi \delta=\omega_{0}^{(\mathcal{P})}-\omega_{p}$ is the laser detuning $\left(\omega_{0}^{(P)}\right.$ is the central pumped resonance frequency in the primary mode family). The integrated dispersion of the primary mode family is defined as $D_{\text {int }}^{(\mathcal{P})}(\mu)=\omega_{\mu}^{(\mathcal{P})}-\omega_{0}^{(\mathcal{P})}-\mu D_{1}^{(\mathcal{P})}$, where $\omega_{\mu}^{(\mathcal{P})}$ indicates the resonance frequency. Similarly, the integrated dispersion of the crossing mode family is $D_{\text {int }}^{(\mathcal{C})}(\mu)=$ $\omega_{\mu}^{(\mathcal{C})}-\omega_{0}^{(\mathcal{C})}-\mu D_{1}^{(\mathcal{C})}$, where $\omega_{\mu}^{(\mathcal{C})}$ is the resonance frequency and $D_{1}^{(\mathcal{C})} / 2 \pi$ indicates the free spectral range of the waveform in the crossing mode family. Therefore, the resonance frequency mismatch between the primary and the crossing mode families is $\Delta(\mu)=\omega_{0}^{(\mathcal{C})}-\omega_{0}^{(\mathcal{P})}+\mu\left(D_{1}^{(\mathcal{C})}-D_{1}^{(\mathcal{P})}\right)$. In addition, $G$ is the linear coupling strength. The singlephoton Kerr frequency shift through self-phase modulation is defined as $g^{(\mathcal{P})}=\left[\hbar\left(\omega_{0}^{(\mathcal{P})}\right)^{2} c n_{2}\right] /\left[\left(n_{0}^{(\mathcal{P})}\right)^{2} V_{\text {eff }}^{(\mathcal{P})}\right]$ and $g^{(\mathcal{C})} \propto\left(1 / V_{\text {eff }}^{(\mathcal{C})}\right)$, where $V_{\text {eff }}^{(\mathcal{P})}$ and $V_{\text {eff }}^{(\mathcal{C})}$ are effective nonlinear mode volumes. The single-photon Kerr frequency shift through cross-phase modulation is given by $g^{(\mathcal{C}-P)} \propto\left(1 / V_{\mathrm{eff}}^{(\mathcal{C}-P)}\right)$, where $V_{\mathrm{eff}}^{(\mathcal{C}-P)}$ is the effective nonlinear mode overlapped volume. The operator $\mathcal{F}[]_{\mu}$ indicates the $\mu$ th frequency component of the Fourier series, and $\delta_{\mu 0}^{\prime}$ is the Kronecker delta. The external pump power coupled to the central mode $(\mu=0)$ is denoted as $\left|s_{\text {in }}\right|^{2}$, and the coupling rate is $\kappa_{\mathrm{ex}}$.

The avoided mode crossing occurs around a mode that satisfies $D_{\text {int }}^{(\mathcal{P})}(\mu) \approx D_{\text {int }}^{(\mathcal{C})}(\mu)+\Delta(\mu)$, or simply $D_{\text {int }}^{(\mathcal{P})} \approx \Delta$ if having $\Delta \gg D_{\text {int }}^{(\mathcal{C})}$ [i.e., in the frame of $D_{\text {int }}^{(\mathcal{P})}(\mu)$, the dispersion of the crossing mode family is almost a slope defined by $\Delta(\mu)$ ]. Nevertheless, the cavity soliton is supported in a range of the laser detuning $(2 \pi \delta)$ and is dispersionless. Therefore, the phase matching between the soliton and the wave in a crossing mode $\left(\mu_{c}\right)$ is $2 \pi \delta+\Delta\left(\mu_{c}\right)=0$.

Switching off the linear coupling $(G=0)$ and the crossphase modulation $\left(g^{(\mathcal{C}-P)}=0\right)$ makes Eq. (A1a) a standard single set of coupled-mode equations (i.e., a standard LLE), where DKS are supported and will follow an analytical solution if $2 \pi \delta \gg \kappa^{(\mathcal{P})}$. In the weak-coupling condition $2 \pi \delta \gg G$, one can assume that DKS remain supported and undepleted. In this way, Eq. (A1b) can be directly solved, neglecting nonlinear terms, to be

$$
\tilde{A}_{\mu}^{(\mathcal{C})}=\frac{i G}{\mathcal{D}(\mu)}\left[e^{(\mathcal{D}(\mu) t)}+1\right] \tilde{A}_{\mu}^{(\mathcal{P})}
$$

where $\mathcal{D}(\mu) \approx-\left(\kappa^{(\mathcal{C})} / 2\right)+i[2 \pi \delta+\Delta(\mu)]$ represents a complex phase mismatch between the soliton and the waveform in the crossing mode family. This solution in return gives rise to a response effect to the dynamics of DKS in the primary mode family where a modified single 
set of coupled wave equations (or a single LLE-like equation) can be derived as (the cross-phase modulation is neglected)

$$
\begin{aligned}
\frac{\partial \tilde{A}_{\mu}^{(\mathcal{P})}(t)}{\partial t}= & \left(-\frac{\kappa^{(\mathcal{P})}}{2}+i(2 \pi \delta)+i D_{\mathrm{int}}^{(\mathcal{P})}(\mu)\right) \tilde{A}_{\mu}^{(\mathcal{P})} \\
& +\tilde{R}_{c}(\mu) \tilde{A}_{\mu}^{(P)}-i g \mathcal{F}\left[\left|A^{(P)}\right|^{2} A^{(P)}\right]_{\mu} \\
& +\delta_{\mu 0}^{\prime} \sqrt{\kappa_{\mathrm{ex}}} s_{\mathrm{in}},
\end{aligned}
$$

where $\tilde{R}_{c}(\mu)=\left(G^{2} / \mathcal{D}(\mu)\right)\left[e^{(\mathcal{D}(\mu) t)}+1\right]$ is named the intermode response whose bandwidth is determined by the loss rate $\kappa^{(\mathcal{C})}$. If $\kappa^{(\mathcal{C})} \ll D_{1}^{(\mathcal{P})}$, only one comb mode is to interact with the intermode response, resulting in a spike in the comb envelope. Physically, the complex amplitude of $\tilde{R}_{c}(\mu)$ indicates that intermode interactions will induce not only a deviation on the dispersion profile (i.e., the imaginary part) in the soliton-filling mode family, as previously studied [40], but equally important, an additional loss channel (i.e., the real part). The extra power loss on DKS will directly degrade the soliton existence range, in particular, the upper boundary that defines the critical power balance between the loss and the pump-induced gain. Equation (A3) can equivalently address the dynamics of DKS in the presence of intermode interactions, including the intermode breathing. Nevertheless, if there is more than one mode satisfying the phase-matching condition, the LLE-like equation can be flexibly extended by simply including several intermode responses.

\section{APPENDIX B: MICRORESONATOR PLATFORMS}

The $\mathrm{MgF}_{2}$ crystalline resonator has a FSR $D_{1}^{(\mathcal{P})}=$ $2 \pi \times 14.094 \mathrm{GHz}$. The $Q$ factor is $\sim 10^{9}$ (intrinsic cavity decay rate $\kappa_{0}^{(\mathcal{P})}=2 \pi \times 80 \mathrm{kHz}$, intrinsic finesse $\left.\sim 1.7 \times 10^{5}\right)$. The second-order dispersion component $\left(D_{2}^{(\mathcal{P})}\right)$ at the pump wavelength (1553 nm) is $D_{2}^{(\mathcal{P})} \approx 2 \pi \times 1.96 \mathrm{kHz}$, while the third-order component is $D_{3}^{(\mathcal{P})}=\mathcal{O}(2 \pi \times 1 \mathrm{~Hz})$. The pump laser (fiber laser, wavelength $1553 \mathrm{~nm}$; short-term linewidth $10 \mathrm{kHz}$ ) is amplified between 20 and $450 \mathrm{~mW}$ and evanescently coupled to the resonator with a tapered optical fiber, which enables the coupling to be adjusted. The loaded linewidth $\kappa^{(\mathcal{P})}=\kappa_{\mathrm{ex}}+\kappa_{0}^{(\mathcal{P})}$ is retrieved by measuring the $\mathcal{C}$-resonance linewidth in the system's response (when no solitons are present in the cavity); the associated coupling coefficient $\left(\kappa_{\mathrm{ex}} / \kappa^{(\mathcal{P})}\right)$ is measured in the range $0.45-0.62$.

The $\mathrm{Si}_{3} \mathrm{~N}_{4}$ integrated microring resonator has a FSR of $\sim 1 \mathrm{THz}$ and the intrinsic $Q$ factor is $\sim 10^{6}$. The resonance intrinsic linewidth is $\kappa_{0}^{(\mathcal{P})} /(2 \pi)=100-150 \mathrm{MHz}$. Such resonators are fabricated using the photonic Damascene process [38]. The ring waveguide cross section has a height of $\sim 850 \mathrm{~nm}$ and a width of $\sim 1450 \mathrm{~nm}$. The dispersion

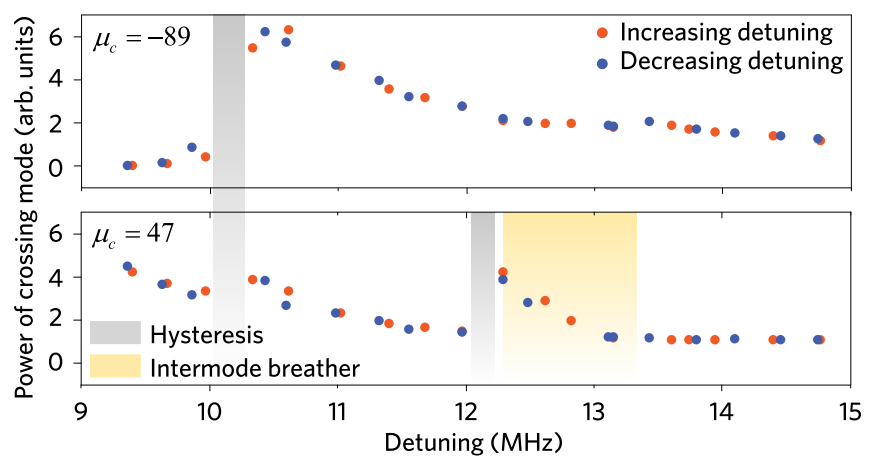

FIG. 5. Power transition in the $\mathrm{MgF}_{2}$ resonator. Measured power traces of power-enhanced modes $\mu_{c}=47$ and $\mu_{c}=-89$, where grayed shading areas mark the power transition and the yellow shading area marks the breathing that is associated with the mode $\mu_{c}=47$.

components at the pump wavelength $(1550 \mathrm{~nm})$ are $D_{2}^{(\mathcal{P})} \approx$ $2 \pi \times 25 \mathrm{MHz}$ and $D_{3}^{(\mathcal{P})}=\mathcal{O}(2 \pi \times 1 \mathrm{MHz})$.

The simulation in Fig. 1 is based on realistic parameters of the $\mathrm{MgF}_{2}$ resonator, whereas the decay rate $\kappa^{(\mathcal{C})}=\kappa^{(\mathcal{P})}$, the linear coupling strength $G=8 \times \kappa^{(\mathcal{P})}$, the FSR mismatch is $D_{1}^{(\mathcal{C})}-D_{1}^{(\mathcal{P})}=2 \pi \times 12 \mathrm{MHz}$, the difference of the central resonance frequency is $\omega_{0}^{(\mathcal{C})}-\omega_{0}^{(\mathcal{P})}=$ $2 \pi \times-600 \mathrm{MHz}$, and the effective nonlinear mode volume is $V_{\text {eff }}^{(\mathcal{P})}=V_{\text {eff }}^{(\mathcal{C})}=2.5 \times 10^{-12} \mathrm{~m}^{3}$. The cross-phase modulation is neglected in the simulation. In addition, the simulation accounts for 1024 modes for both the primary and the crossing mode families.

\section{APPENDIX C: POWER TRANSITION IN $\mathrm{MgF}_{2}$ RESONATOR}

The power-enhanced modes in the $\mathrm{MgF}_{2}$ resonator are also monitored (see Fig. 5). While several power transitions are observed in modes $\mu_{c}=47$ and $\mu_{c}=-89$, the hysteresis is not obvious.

[1] N. Akhmediev and A. Ankiewicz, in Dissipative Solitons: From Optics to Biology and Medicine, Lecture Notes in Physics (Springer, New York, 2008).

[2] C. Bao, W. Chang, C. Yang, N. Akhmediev, and S. T. Cundiff, Observation of Coexisting Dissipative Solitons in a Mode-Locked Fiber Laser, Phys. Rev. Lett. 115, 253903 (2015).

[3] G. Herink, F. Kurtz, B. Jalali, D. R. Solli, and C. Ropers, Real-Time Spectral Interferometry Probes the Internal Dynamics of Femtosecond Soliton Molecules, Science 356, 50 (2017).

[4] K. Krupa, K. Nithyanandan, U. Andral, P. Tchofo-Dinda, and P. Grelu, Real-Time Observation of Internal Motion within Ultrafast Dissipative Optical Soliton Molecules, Phys. Rev. Lett. 118, 243901 (2017). 
[5] F. Leo, L. Gelens, P. Emplit, M. Haelterman, and S. Coen, Dynamics of One-Dimensional Kerr Cavity Solitons, Opt. Express 21, 9180 (2013).

[6] P. Parra-Rivas, D. Gomila, M. A. Matías, S. Coen, and L. Gelens, Dynamics of Localized and Patterned Structures in the Lugiato-Lefever Equation Determine the Stability and Shape of Optical Frequency Combs, Phys. Rev. A 89, 043813 (2014).

[7] C. Godey, I. V. Balakireva, A. Coillet, and Y. K. Chembo, Stability Analysis of the Spatiotemporal Lugiato-Lefever Model for Kerr Optical Frequency Combs in the Anomalous and Normal Dispersion Regimes, Phys. Rev. A 89, 063814 (2014).

[8] E. Fermi, J. Pasta, and S. Ulam, Los Alamos Scientific Laboratory, Technical Report No. LA-1940, 1955, https:// www.osti.gov/scitech/servlets/purl/4376203.

[9] J. M. Dudley, F. Dias, M. Erkintalo, and G. Genty, Instabilities, Breathers and Rogue Waves in Optics, Nat. Photonics 8, 755 (2014).

[10] P. Grelu and N Akhmediev, Dissipative Solitons for ModeLocked Fiber Lasers, Nat. Photonics 6, 84 (2012).

[11] C. Bao, J. A. Jaramillo-Villegas, Y. Xuan, D. E. Leaird, M. Qi, and A. M. Weiner, Observation of Fermi-Pasta-Ulam Recurrence Induced by Breather Solitons in an Optical Microresonator, Phys. Rev. Lett. 117, 163901 (2016).

[12] M. Yu, J. K. Jang, Y. Okawachi, A. G. Griffith, K. Luke, S. A. Miller, X. Ji, M. Lipson, and A. L. Gaeta, Breather Soliton Dynamics in Microresonators, Nat. Commun. 8, 14569 (2017).

[13] E. Lucas, M. Karpov, H. Guo, M. L. Gorodetsky, and T. J. Kippenberg, Breathing Dissipative Solitons in Optical Microresonators, Nat. Commun. 8, 736 (2017).

[14] T. Herr, V. Brasch, J. D. Jost, C. Y. Wang, N. M. Kondratiev, M. L. Gorodetsky, and T. J. Kippenberg, Temporal Solitons in Optical Microresonators, Nat. Photonics 8, 145 (2014).

[15] V. Brasch, M. Geiselmann, T. Herr, G. Lihachev, M. H. P. Pfeiffer, M. L. Gorodetsky, and T. J. Kippenberg, Photonic Chip-Based Optical Frequency Comb Using Soliton Cherenkov Radiation, Science 351, 357 (2016).

[16] X. Yi, Q.-F. Yang, K. Y. Yang, M.-G. Suh, and K. Vahala, Soliton Frequency Comb at Microwave Rates in a High-Q Silica Microresonator, Optica 2, 1078 (2015).

[17] W. Liang, D. Eliyahu, V. S. Ilchenko, A. A. Savchenkov, A. B. Matsko, D. Seidel, and L. Maleki, High Spectral Purity Kerr Frequency Comb Radio Frequency Photonic Oscillator, Nat. Commun. 6, 7957 (2015).

[18] P.-H. Wang, J. A. Jaramillo-Villegas, Y. Xuan, X. Xue, C. Bao, D. E. Leaird, M. Qi, and A. M. Weiner, Intracavity Characterization of Micro-Comb Generation in the SingleSoliton Regime, Opt. Express 24, 10890 (2016).

[19] C. Joshi, J. K. Jang, K. Luke, X. Ji, S. A. Miller, A. Klenner, Y. Okawachi, M. Lipson, and A. L. Gaeta, Thermally Controlled Comb Generation and Soliton Modelocking in Microresonators, Opt. Lett. 41, 2565 (2016).

[20] Q. Li, T. C. Briles, D. A. Westly, T. E. Drake, J. R. Stone, B. R. Ilic, S. A Diddams, S. B. Papp, and K. Srinivasan, Stably Accessing Octave-Spanning Microresonator Frequency Combs in the Soliton Regime, Optica 4, 193 (2017).

[21] M. H. P. Pfeiffer, C. Herkommer, J. Liu, H. Guo, M. Karpov, E. Lucas, M. Zervas, and T. J. Kippenberg,
Octave-Spanning Dissipative Kerr Soliton Frequency Combs in $\mathrm{Si}_{3} \mathrm{~N}_{4}$ Microresonators, Optica 4, 684 (2017).

[22] M.-G. Suh, Q.-F. Yang, K.-Y. Yang, X. Yi, and K. J. Vahala, Microresonator Soliton Dual-Comb Spectroscopy, Science 354, 600 (2016).

[23] M. Yu, Y. Okawachi, A. G. Griffith, N. Picqué, M. Lipson, and A. L. Gaeta, Silicon-Chip-Based Mid-Infrared Dual-Comb Spectroscopy, arXiv:1610.01121.

[24] J. D. Jost, T. Herr, C. Lecaplain, V. Brasch, M. H. P. Pfeiffer, and T. J. Kippenberg, Counting the Cycles of Light Using a Self-Referenced Optical Microresonator, Optica 2, 706 (2015).

[25] V. Brasch, E. Lucas, J. D. Jost, M. Geiselmann, and T. J. Kippenberg, Self-Referenced Photonic Chip Soliton Kerr Frequency Comb, Light Sci. Appl. 6, e16202 (2017).

[26] P. Marin-Palomo et al., Microresonator-Based Solitons for Massively Parallel Coherent Optical Communications, Nature (London) 546, 274 (2017).

[27] P. Trocha et al., Ultrafast optical ranging using microresonator soliton frequency combs, arXiv:1707.05969.

[28] M.-G. Suh and K. Vahala, Soliton microcomb range measurement, arXiv:1705.06697.

[29] D. T. Spencer et al., An integrated-photonics opticalfrequency synthesizer, arXiv:1708.05228.

[30] I. V. Barashenkov and Y. S. Smirnov, Existence and Stability Chart for the ac-Driven, Damped Nonlinear Schrödinger Solitons, Phys. Rev. E 54, 5707 (1996).

[31] L. A. Lugiato and R. Lefever, Spatial Dissipative Structures in Passive Optical Systems, Phys. Rev. Lett. 58, 2209 (1987).

[32] Y. K. Chembo and N. Yu, Modal Expansion Approach to Optical-Frequency-Comb Generation with Monolithic Whispering-Gallery-Mode Resonators, Phys. Rev. A 82, 033801 (2010).

[33] S. Coen, H. G. Randle, T. Sylvestre, and M. Erkintalo, Modeling of Octave-Spanning Kerr Frequency Combs Using a Generalized Mean-Field Lugiato-Lefever Model, Opt. Lett. 38, 37 (2013).

[34] Y. K. Chembo and C. R. Menyuk, Spatiotemporal Lugiato-Lefever Formalism for Kerr-Comb Generation in Whispering-Gallery-Mode Resonators, Phys. Rev. A 87, 053852 (2013).

[35] E. Lucas, H. Guo, J. D. Jost, M. Karpov, and T. J. Kippenberg, Detuning-Dependent Properties and Dispersion-Induced Instabilities of Temporal Dissipative Kerr Solitons in Optical Microresonators, Phys. Rev. A 95, 043822 (2017).

[36] H. Guo, M. Karpov, E. Lucas, A. Kordts, M. H. P. Pfeiffer, V. Brasch, G. Lihachev, V. E. Lobanov, M. L. Gorodetsky, and T. J. Kippenberg, Universal Dynamics and Deterministic Switching of Dissipative Kerr Solitons in Optical Microresonators, Nat. Phys. 13, 94 (2017).

[37] J. Riemensberger, K. Hartinger, T. Herr, V. Brasch, R. Holzwarth, and T. J. Kippenberg, Dispersion Engineering of Thick High-Q Silicon Nitride Ring-Resonators via Atomic Layer Deposition, Opt. Express 20, 27661 (2012).

[38] M. H. P. Pfeiffer, A. Kordts, V. Brasch, M. Zervas, M. Geiselmann, J. D. Jost, and T. J. Kippenberg, Photonic Damascene Process for Integrated High-Q Microresonator Based Nonlinear Photonics, Optica 3, 20 (2016).

[39] T. Carmon, H. G. L. Schwefel, L. Yang, M. Oxborrow, A. D. Stone, and K. J. Vahala, Static Envelope Patterns in 
Composite Resonances Generated by Level Crossing in Optical Toroidal Microcavities, Phys. Rev. Lett. 100, 103905 (2008).

[40] T. Herr, V. Brasch, J. D. Jost, I. Mirgorodskiy, G. Lihachev, M. L. Gorodetsky, and T. J. Kippenberg, Mode Spectrum and Temporal Soliton Formation in Optical Microresonators, Phys. Rev. Lett. 113, 123901 (2014).

[41] A. B. Matsko, W. Liang, A. A. Savchenkov, D. Eliyahu, and L. Maleki, Optical Cherenkov Radiation in Overmoded Microresonators, Opt. Lett. 41, 2907 (2016).

[42] Q.-F. Yang, X. Yi, K.-Y. Yang, and K. J. Vahala, SpatialMode-Interaction-Induced Dispersive Waves and Their Active Tuning in Microresonators, Optica 3, 1132 (2016).

[43] X. Yi, Q.-F. Yang, X. Zhang, K. Y. Yang, X. Li, and K. Vahala, Single-Mode Dispersive Waves and Soliton Microcomb Dynamics, Nat. Commun. 8, 14869 (2017).

[44] M. Karpov, H. Guo, A. Kordts, V. Brasch, M. H. P. Pfeiffer, M. Zervas, M. Geiselmann, and T. J. Kippenberg, Raman SelfFrequency Shift of Dissipative Kerr Solitons in an Optical Microresonator, Phys. Rev. Lett. 116, 103902 (2016).

[45] X. Yi, Q.-F. Yang, K.-Y. Yang, and K. J. Vahala, Theory and Measurement of the Soliton Self-Frequency Shift and
Efficiency in Optical Microcavities, Opt. Lett. 41, 3419 (2016).

[46] S. Wabnitz, Suppression of Interactions in a Phase-Locked Soliton Optical Memory, Opt. Lett. 18, 601 (1993).

[47] G. D'Aguanno and C. R. Menyuk, Nonlinear Mode Coupling in Whispering-Gallery-Mode Resonators, Phys. Rev. A 93, 043820 (2016).

[48] H. A. Haus and W. Huang, Coupled-Mode Theory, Proc. IEEE 79, 1505 (1991).

[49] J. Wiersig, Formation of Long-Lived, Scarlike Modes Near Avoided Resonance Crossings in Optical Microcavities, Phys. Rev. Lett. 97, 253901 (2006).

[50] S. Coen and M. Erkintalo, Universal Scaling Laws of Kerr Frequency Combs, Opt. Lett. 38, 1790 (2013).

[51] A. L. Lugiato, Theory of Optical Bistability, Prog. Opt. 21, 69 (1984).

[52] N. Akhmediev, J. M. Soto-Crespo, and G. Town, Pulsating Solitons, Chaotic Solitons, Period Doubling, and Pulse Coexistence in Mode-Locked Lasers: Complex GinzburgLandau Equation Approach, Phys. Rev. E 63, 056602 (2001).

[53] http://dx.doi.org/10.5281/zenodo.1043981. 